\title{
Freqüência de infecção pelo Mycoplasma hominis e Ureaplasma urealyticum em mulheres inférteis e relação com repercussões clínicas
}

\author{
Frequency of infection with Mycoplasma hominis and Ureaplasma urealyticum in \\ infertile women and clinical repercussions
}

\begin{abstract}
Ivan Araujo Penna ${ }^{1}$, Geraldo Duarte ${ }^{2}$, Rui Alberto Ferriani ${ }^{3}$, Alcyone Artioli Machado ${ }^{4}$,
\end{abstract} Gustavo Salata Romão ${ }^{5}$, Rosana Maria dos Reis ${ }^{6}$, Marcos Dias de Moura ${ }^{7}$

\section{RESUMO}

Objetivos: determinar a freqüência de infecção pelo Mycoplasma hominis e Ureaplasma urealyticum e relacioná-la a variáveis clínicas de mulheres inférteis. Métodos: estudo transversal com 322 pacientes inférteis submetidas à coleta de swab endocervical para pesquisa de Mycoplasma hominis e Ureaplasma urealyticum, de outubro de 2002 a maio de 2004. Todas as pacientes foram submetidas a protocolo básico de investigação clínica e laboratorial da infertilidade. Como controle, utilizou-se série histórica de 51 mulheres não gestantes, previamente pesquisadas quanto aos agentes infecciosos estudados. Resultados: a freqüência de infecção pelo Mycoplasma hominis e Ureaplasma urealyticum foi de 4,9\% nas pacientes inférteis e $13,8 \%$ no grupo controle. Entre as pacientes inférteis observou-se relação entre a presença dos dois patógenos e alterações no resultado da histerossalpingografia (OR: 3,20; IC 95\%: 1,05-9,73), presença de dispareunia (OR: 10,72; IC 95\%: 3,21-35,77) e corrimento vaginal (OR: 8,5; IC 95\%: 2,83-26,02), além de cultura endocervical positiva para Escherichia coli (OR: 16,09; IC 95\%: 4,95-52,25). Conclusão: a taxa de infecção pelo Mycoplasma hominis e Ureaplasma urealyticum é baixa em pacientes inférteis e está associada a seqüelas reprodutivas tardias.

PALAVRAS-CHAVE: Mycoplasma hominis; Ureaplasma urealyticum; Infertilidade e Escherichia coli; Histerossalpingografia

\section{ABSTRACT}

Purpose: to determine the frequency of Mycoplasma hominis and Ureaplasma urealyticum infection, and relate it to the associated clinical variables of infertile women. Methods: transversal study involving 322 infertile women, submitted to collection of endocervix swab for research of Mycoplasma hominis and Ureaplasma urealyticum infecction, from October 2002 to May 2004. All patients were submitted to a basic infertility investigation protocol. As control, a historical series of 51 non-pregnant women previously investigated as for the studied infectious agents, was used. Results: the frequency of Mycoplasma hominis and Ureaplasma urealyticum infection was $4.9 \%$ in the infertile women and $13.8 \%$ in the control group. Among the infertile patients, a relationship between the presence of the two agents and changes in the histerosalpingography result (OR: 3.20; IC 95\%: 1.05-9.73), presence of dyspareunia (OR: 10.72; IC 95\%: 3.21-35.77) and vaginal discharge (OR: 8.5; IC 95\%: 2.83-26.02), besides endocervical culture positive for Escherichia coli (OR: 6.09; IC 95\%: 4.95-52.25) was observed. Conclusion: Mycoplasma hominis and Ureaplasma urealyticum infection rate is low in infertile patients and is associated with reproductive sequels.

KEYWORDS: Mycoplasma hominis; Ureaplasma urealyticum; Infertility and Escherichia coli; Hysterosalpingography

Departamento de Ginecologia e Obstetrícia da Faculdade de Medicina de Ribeirão Preto da Universidade de São Paulo

1 Pós-graduando

2,3,6,7 Docentes

4 Docentes do Departamento de Clinica Médica

5 Médico Assistente do Hospital das Clínicas de Ribeirão Preto

Correspondência: Rui Alberto Ferriani

Av. Bandeirantes, 3900 - 14049-900 - Ribeirão Preto - SP - Telefone: (16) 602-2818 - e-mail: raferria@fmrp.usp.br

Recebido em: 3/11/2004

Aceito com modificações em: 17/1/2005 
Introdução

$\mathrm{Na}$ rotina de investigação do casal infértil a pesquisa de infecções genitais sempre foi cercada de controvérsias. Dados da literatura fundamentam o impacto negativo dessas infecções sobre as variáveis ligadas à reprodução, mas nota-se grande variação na incidência destas alterações ${ }^{1}$. As doenças sexualmente transmissiveis podem causar alterações no aparelho genital masculino ou feminino, potencialmente predispondo a seqüelas. Atualmente, o microrganismo mais associado à infertilidade feminina é a Chlamydia trachomatis, com freqüência que varia de 30 a $50 \%$ dos $\operatorname{casos}^{2,3}$.

A infecção pelos Mycoplasma também leva a seqüelas no sistema reprodutivo, principalmente na tuba uterina e na capacidade de migração dos espermatozóides. A sua freqüência na população de pacientes inférteis não é homogênea na literatura, variando entre 2 e $23 \%$. A ampla variabilidade nestas taxas depende de fatores populacionais e das diferentes técnicas diagnósticas empregadas, dificultando traçar um perfil clínico característico ${ }^{1,2,4}$.

A avaliação diagnóstica rotineira da infecção por Mycoplasma hominis e Ureaplasma urealyticum em casos de infertilidade é questionada por alguns serviços, em virtude de se instituir o tratamento profilático com antibióticos antes da realização de métodos invasivos de investigação do casal infértil ${ }^{5}$. Como a freqüência de infecção pelos micoplasmas é variável, o presente estudo objetivou determinar a freqüência de Mycoplasma hominis e Ureaplasma urealyticum em pacientes inférteis atendidas em centro terciário de infertilidade, avaliando as variáveis clínicas associadas a essa infecção e discutindo os custos inerentes ao processo diagnóstico comparados aos custos do tratamento profilático.

\section{Pacientes e Métodos}

Estudo transversal no qual foram incluídas 322 pacientes inférteis atendidas em centro terciário de infertilidade conjugal (Hospital das Clínicas da Faculdade de Medicina de Ribeirão Preto da Universidade de São Paulo, FMRP-USP). No período de outubro de 2002 a maio de 2004 foi instituído o procedimento rotineiro de coleta de swab endocervical e pesquisa de Mycoplasma hominis e Ureaplasma urealyticum, para todas as pacientes novas do ambulatório de esterilidade conjugal. Como grupo controle, foram utilizados os dados de série histórica de 51 mulheres sem quei- xas ginecológicas e não gestantes, da mesma região demográfica (outra amostra populacional, cuja freqüência de infecção por micoplasmas foi descrita anteriormente ${ }^{6}$. O presente estudo foi aprovado pelo Comitê de Ética em Pesquisa do Hospital das Clínicas da FMRP-USP.

Os dados foram levantados a partir das informações dos prontuários médicos das pacientes incluídas. Foram critérios básicos de inclusão no grupo de estudo: pacientes encaminhadas da rede básica de saúde com tempo de tentativa de concepção superior a dois anos ou causa de infertilidade pré-estabelecida e idade materna inferior a 38 anos e submetidas a todos os procedimentos do protocolo de investigação do ambulatório, com os dados completos no prontuário. No período pesquisado, houve exclusão de 40 prontuários por dados incompletos $(11,04 \%)$.

Todas as pacientes do grupo de estudo foram submetidas ao protocolo básico de investigação dos fatores causais de infertilidade conjugal ${ }^{5}$, utilizando avaliação clínica e exame físico, espermograma e espermocultura do parceiro, swab endocervical, histerossalpingografia, histeroscopia, ultrasonografia transvaginal, dosagens de hormônio folículo-estimulante, hormônio estimulador da tiróide, prolactina e laparoscopia, quando indicada.

Para a detecção do Mycoplasma hominis e Ureaplasma urealyticum no swab endocervical utilizou-se o kit Mycofast Screening Evolution $2^{\circledast}$ (International Microbio, Signes, France). A positividade relaciona-se com a propriedade do Ureaplasma e Mycoplasma de metabolizarem respectivamente uréia e arginina, promovendo marcação quantitativa colorimétrica. O material colhido é semeado em bandeja com duas fileiras de tubos de ensaio contendo os dois substratos para o metabolismo. A quantificação é feita a partir da contagem do número de tubos que apresentarem mudança de coloração. A sensibilidade do método é de $98,2 \%$ e a especificidade de $100 \%{ }^{7,8}$.

Foi avaliado se a presença de infecção por Mycoplasma hominis e Ureaplasma urealyticum relacionava-se à idade, tempo de infertilidade, causa de infertilidade, tipo de infertilidade (primária ou secundária), alterações na histerossalpingografia e histeroscopia, presença de outros microrganismos na cultura endocervical, queixa de dispareunia e corrimento genital na primeira consulta.

Para a análise de custos, levou-se em conta a cotação média da azitromicina 1 grama a partir do valor obtido de três diferentes farmácias da cidade de Ribeirão Preto, sendo o custo calculado a partir de um valor teórico de tratamento de todas as pacientes, independente de terem ou não infecção. O custo atribuído ao diagnóstico foi esti- 
mado levando-se em conta apenas o valor dos kits para investigação.

Para a análise estatística, cujas variáveis apresentaram distribuição normal, tais como a idade e o tempo de tentativa de gravidez, utilizouse o teste $t$ de Student não pareado, considerando o nível de significância de $\mathrm{p} \leq 0,05$. Para verificar as associações foram utilizados o teste do $\chi^{2}$ e regressão multivariada, utilizando odds ratio (OR) e intervalo de confiança (IC) de 95\%. O software utilizado foi o Graph-pad Prisma, versão 3.0 (San Diego, CA, USA).

\section{Resultados}

A freqüência de positividade para Mycoplasma hominis e Ureaplasma urealyticum foi de $4,9 \%$ para as mulheres com infertilidade. $\mathrm{Na}$ Tabela 1 essa freqüência do grupo de estudo é comparada à freqüência de outra amostra da mesma população sem infertilidade ${ }^{6}$, no qual se observa freqüência de 13,8\% (Tabela 1). Em relação ao grupo de estudo (pacientes inférteis), a taxa de positividade para estes micro-organismos não foi relacionada com idade $(30,3 \pm 3,24$ anos $)$, média de tempo de tentativa de concepção $(4,5 \pm 2,36$ anos) e taxa de gravidez (31\%) obtida após terapêutica específica (Tabela 2). Também não foi observada associação entre as várias causas de infertilidade e a positividade do teste para Mycoplasma hominis e/ou Ureaplasma urealyticum nessas pacientes (Tabela 3).

Observou-se associação significante entre a presença dos dois patógenos estudados e algumas características clinicas nas pacientes inférteis, tais como: alteração no resultado da histerossalpingografia $(n=5 ; 31,25 \%$; OR: 3,20; IC 95\%: 1,05-9,73), presença de dispareunia $(n=5$; 31,25\%; OR: 10,72; IC 95\%: 3,21-35,77) e corrimento genital ( $\mathrm{n}=6$; 37,5\%; OR: 8,5; IC 95\%: 2,83$26,02)$ como queixa na primeira consulta e cultura endocervical positiva para Escherichia coli (n=6; 37,5\%; OR: 6,09; IC 95\%: 4,95-52,25). Não se observou associação da presença de micoplasmas com alterações uterinas na histeroscopia ( $\mathrm{n}=2 ; 12,5 \%$, OR: 1,09; IC 95\%: 0,21$9,20)$ e com o tipo de infertilidade $(n=2 ; 12,5 \%$; OR: 0,55; IC 95\%: 0,20-1,50) (Tabela 4).

Tabela 1 - Freqüência dos diagnósticos de Mycoplasma hominis elou Ureaplasma urealyticum no conteúdo vaginal de pacientes inférteis e do grupo controle.

\begin{tabular}{|c|c|c|c|c|c|c|c|c|}
\hline \multirow[t]{3}{*}{ Agente etiológico } & \multicolumn{4}{|c|}{ Inférteis } & \multicolumn{4}{|c|}{ Controle } \\
\hline & \multicolumn{2}{|c|}{ Positivo } & \multicolumn{2}{|c|}{ Negativo } & \multicolumn{2}{|c|}{ Positivo } & \multicolumn{2}{|c|}{ Negativo } \\
\hline & $\mathrm{n}$ & $\%$ & $\mathrm{n}$ & $\%$ & $\mathrm{n}$ & $\%$ & $\mathrm{n}$ & $\%$ \\
\hline Mycoplasma hominis & 5 & 1,5 & 316 & 98,1 & 1 & 11,8 & 50 & 98 \\
\hline Ureaplasma urealyticum & 10 & 3,1 & 311 & 96,5 & 6 & 11,8 & 45 & 88,2 \\
\hline Ambos & 1 & 0,3 & 321 & 99,7 & 0 & 0 & 51 & 100 \\
\hline Total & 16 & 4,9 & 306 & 95,1 & 7 & 13,8 & 44 & 86,2 \\
\hline
\end{tabular}

Valores absolutos e porcentagem.

Tabela 2 - Características clínicas das pacientes inférteis estudadas, de acordo com a positividade para Mycoplasma hominis e Ureaplasma urealyticum.

\begin{tabular}{lccc}
\hline & M. hominis & U. urealyticum & Negativo \\
& $\overline{\mathbf{x}} \pm$ DP & $\overline{\mathbf{x}} \pm \mathbf{D P}$ & $\overline{\mathbf{x}} \pm \mathbf{D P}$ \\
\hline Idade (anos) & $30,3 \pm 3,24$ & $29,4 \pm 3,87$ & $31,5 \pm 4,37$ \\
Tempo de tentativa (anos) & $4,5 \pm 2,36$ & $4,09 \pm 2,67$ & $4,78 \pm 3,10$ \\
Taxa de gravidez (\%) & 31 & 35 & 30 \\
\hline
\end{tabular}

$\overline{\mathrm{x}}=$ média; DP $= \pm$ desvio padrão; teste $t$ de Student e teste $\chi^{2}$.

Não houve diferença significativa.
Tabela 3 - Frequêencia das causas de infertilidade no grupo de estudo, de acordo com a positividade para Mycoplasma hominis e Ureaplasma urealyticum.

Myco e/ou Ureaplasma Myco e/ou Ureaplasma

Positivo Negativo

\begin{tabular}{|c|c|c|c|c|}
\hline Fatores & $\mathrm{n}$ & $\%$ & $\mathrm{n}$ & $\%$ \\
\hline Tubária & 4 & 25,0 & 46 & 15,0 \\
\hline Peritoneal & 2 & 12,5 & 25 & 8,2 \\
\hline Ovulatória & 2 & 12,5 & 23 & 7,5 \\
\hline Masculina & 5 & 31,2 & 127 & 41,5 \\
\hline $\begin{array}{r}\text { Duas causas } \\
\text { associadas }\end{array}$ & 3 & 0,9 & 77 & 25,1 \\
\hline $\begin{array}{l}\text { Esterilidade sem } \\
\text { causa aparente }\end{array}$ & 0 & 0 & 8 & 2,8 \\
\hline Total & 16 & 100 & 306 & 100 \\
\hline
\end{tabular}


Tabela 4 - Razão de chance de positividade para Mycoplasma hominis e/ou Ureaplasma urealyticum no swab endocervical, de acordo com algumas características clínicas e laboratoriais encontradas nas pacientes inférteis.

\begin{tabular}{lllccc}
\hline & $\mathbf{n}$ & $\%$ & Odds ratio & IC 95\% & p \\
\hline Infertilidade primária & 2 & 12,5 & 0,55 & $0,20-1,50$ & 0,242 \\
Histerossalpingografia alterada & 5 & 31,2 & 3,20 & $1,05-9,73^{*}$ & $<0,05$ \\
Histeroscopia alterada & 2 & 12,5 & 1,09 & $0,21-9,20$ & 0,565 \\
Dispareunia & 5 & 31,2 & 10,72 & $3,21-35,77^{*}$ & $<0,05$ \\
Corrimento genital & 6 & 37,5 & 8,5 & $2,83-26,02^{*}$ & $<0,05$ \\
Swab positivo para Escherichia coli & 6 & 37,5 & 16,09 & $4,95-52,25^{*}$ & $<0,05$ \\
\hline
\end{tabular}

*Intervalo de confiança (IC) de $95 \%$ foi estatisticamente significante.

$\mathrm{Na}$ avaliação de custos, a investigação diagnóstica de todas as pacientes inférteis custou $R \$$ $26.049,60$, enquanto o tratamento a todas elas custaria $\mathrm{R} \$ 6.440,00$.

\section{Discussão}

As infecções genitais podem ser consideradas como uma das principais causas de esterilidade, uma vez que provocam seqüelas transitórias ou permanentes nos órgãos relacionados à reprodução, tanto masculinos como femininos ${ }^{2}$. Diferente de décadas passadas, quando a Neisseria gonorrhoeae era o agente etiológico mais conhecido e o agente causal mais freqüente, hoje observa-se que a Chlamydia trachomatis apresenta as maiores taxas de prevalência, variando de 30 a $50 \%$, dependendo da população estudada ${ }^{2}$.

As infecções pelo Mycoplasma hominis, Mycoplasma genitalium e Ureaplasma urealyticum, apesar de menos freqüentes, também podem causar efeitos nocivos à fertilidade e ao sistema gênitourinário $^{1,3}$. No presente estudo, as pacientes inférteis apresentaram menor freqüência de infecção pelo Ureaplasma urealyticum que as pacientes do grupo controle e freqüência semelhante de Mycoplasma. hominis. Apesar de a literatura também corroborar essa diferença, os valores de 4,8 e 23,5\%, respectivamente para Mycoplasma hominis e Ureaplasma urealyticum, apresentados por Rodriguez et al. ${ }^{1}$, são superiores aos valores de 1,5 e $3,1 \%$, respectivamente, encontrados nas pacientes inférteis do presente estudo. Ressalta-se, entretanto, que as pacientes do presente estudo pertencem a um serviço terciário de infertilidade conjugal, e pode ter havido tratamento prévio em serviços de atendimento de menor complexidade.

Na literatura é citado como padrão-ouro para o diagnóstico de Mycoplasma hominis e Ureaplasma urealyticum a reação em cadeia da polimerase (PCR), mas segundo Grattard et al. ${ }^{7}$ a quantificação colorimétrica do metabolismo dos dois microrganismos também possui boa sensibilidade e especificidade, justificando seu uso como método diagnóstico. A PCR, apesar de possuir a melhor especificidade para este diagnóstico, falha por não ser capaz de diferenciar a paciente portadora da infecção daquela simplesmente colonizada.

A associação entre as várias causas de esterilidade diagnosticada entre as mulheres do estudo e a presença da infecção pelo Mycoplasma hominis e Ureaplasma urealyticum não mostrou diferenças significativas. Ainda assim, a literatura relaciona essas infecções às causas masculinas e tubárias ${ }^{9,10}$. Provavelmente isso ocorreu devido à baixa freqüência do diagnóstico na população estudada e por não ter sido avaliado o fator masculino.

Apesar de não ter havido associação entre as causas tubárias de infertilidade e a infecção pelo Mycoplasma hominis e Ureaplasma urealyticum, observou-se associação entre a presença de alterações da histerossalpingografia e a infecção por estes microrganismos. Torna-se dificil com apenas um estudo determinar a ligação de causa e efeito entre a infecção por Ureaplasma urealyticum e as doenças obstrutivas tubárias ${ }^{1}$, pois apesar de este microrganismo estar relacionado à corioamionite e à hipertermia pós-parto, não está bem caracterizado que o mesmo possa levar a doenças obstrutivas tubárias. A literatura relaciona Mycoplasma hominis e o Mycoplasma genitalium como causas de doença inflamatória pélvica (DIP) e conseqüentes seqüelas, sendo a taxa de ocorrência cerca de $22 \%$ dos casos de acometimento pelo Mycoplasma genitalium ${ }^{11}$.

Em contraponto às lesões observadas na histerosalpingografia, não se notaram alterações tanto na histeroscopia quanto na ultra-sonografia no grupo de pacientes infectadas. Por isso, acredita-se serem necessários outros estudos com maior enfoque das patologias anatômicas da tuba uterina e a infecção genital por Ureaplasma urealyticum, como os de Xu et al. ${ }^{12}$.

Também foi observada associação entre 
queixas de corrimento vaginal e dispareunia na primeira consulta no ambulatório de esterilidade e a infecção. Tais dados não foram encontrados na literatura, mas Judlin ${ }^{3}$ determina que 2/3 dos casos de vaginose bacteriana estão associados à presença dos dois patógenos. Em 2001, van Belkum et al. ${ }^{4}$ mostraram associação de até $79 \%$ entre Trichomonas vaginalis e Mycoplasma hominis, o mesmo não sendo observado na presença de coinfecção com Candida albicans. No presente estudo, observou-se associação entre a infecção por Escherichia coli e a infecção por Mycoplasma hominis e Ureaplasma urealyticum.

Em relação aos custos da investigação para Mycoplasma hominis e Ureaplasma urealyticum, observou-se que a realização rotineira desses testes acarretou custo significativo, que deve ser adicionado aos custos totais de propedêutica de um casal infértil. A nosso ver, acrescentar-se esses custos não é relação efetiva, já que o tratamento, se fosse aplicado sistematicamente, reduziria o custo a patamares quatro vezes menores que a investigação sistemática. Essa abordagem, entretanto, pode acarretar outros custos adicionais, não computados nesse trabalho, como alívio de efeitos colaterais e efeitos decorrentes de alterações da flora vaginal.

No presente estudo, a incidência da infecção por Mycoplasma hominis e Ureaplasma urealyticum em pacientes atendidas no ambulatório de esterilidade foi baixa, ao mesmo tempo em que não houve variação nas taxas de gravidez em relação ao grupo controle. Desta forma, a investigação rotineira de Mycoplasma hominis e Ureaplasma urealyticum em todas as mulheres inférteis não está indicada, em virtude da baixa incidência e pelo fato de serem estes agentes sensiveis a antibióticos rotineiramente utilizados previamente a procedimentos invasivos da mulher infértil, tais como a histeroscopia e fertilização in vitro. Nas pacientes que apresentam queixas de dispareunia e/ou corrimento genital, alterações à histerossalpingografia e portadoras de infecções por Echerichia coli, a possibilidade de achado de positividade destes microrganismos aumenta, podendo suscitar ilações quanto a um possivel grupo de risco.

\section{Referências}

1. Rodriguez R, Hernández R, Fuster F, Torres A, Prieto $\mathrm{P}$, Alberto J. Infección genital y esterilidad. Enferm Infecc Microbiol Clin. 2001; 19:261-6.

2. Horner P, Thomas B, Gilroy CB, Egger M, TaylorRobinson D. Role of Mycoplasma genitalium and Ureaplasma urealyticum in acute and chronic nongonococcal urethritis. Clin Infect Dis. 2001; 32:995-1003.

3. Judlin P. Genital mycoplasmas. Gynecol Obstet Fertil 2003; 31:954-9.

4. Van Belkum A, van der Schee V, van der Meijden WI, Verbrugh HA, Sluiters HJ. A clinical study on the association of Trichomonas vaginalis and Mycoplasma hominis infections in women attending a sexually transmitted disease (STD) outpatient clinic. FEMS Immunol Med Microbiol. 2001; 32:27-32.

5. Ferreira RA, Navarro PAAS. Técnicas de reprodução assistida. In: Ferriani RA, Navarro PAAS, editores. Protocolos de Condutas em Infertilidade Conjugal. $1^{\text {a }}$ ed. São Paulo: Conexão Brasil; 2004. p. 65.

6. Machado AA, Zorzi AR, Gléria AEA, Donadi EA. Frequency of Mycoplasma hominis and ureaplasma urealyticum infections in women with systemic lupus erythematosus. Rev Soc Bras Med Trop. 2001; 34:243-7.

7. Grattard F, Soleihac B, De Barbeyrac B, Bebear C, Seffert P, Pozzetto B. Epidemiologic and molecular investigations of genital mycoplasmas from women and neonates at delivery. Pediatr Infect Dis J. 1995; $14: 853-8$.

8. Duarte G, Machado AA, Beitune P, et al. Infecçäo genital feminina por Mycoplasma hominis e Ureaplasma urealyticum e sua relaçäo com a gravidez e a infecçäo pelo HIV-1. DST J Bras Doenças Sex Transm. 1998; 10:5-8.

9. Levy R, Layani-Milon MP, Giscard D'Estaing S, et al. Screening for Chlamydia trachomatis and Ureaplasma urealyticum infection in semen from asymptomatic male partners of infertile couples prior to in vitro fertilization. Int J Androl. 1999; 22:113-8.

10.Jensen JS. Mycoplasma genitalium: the aetiological agent of urethritis and other sexually transmitted diseases. J Eur Acad Dermatol Venereol. 2004; 18:1-11.

11.Xu C, Sun GF, Zhu YF, Wang YF. The correlation of Ureaplasma urealyticum infection with infertility. Andrologia. 1997; 29:219-26. 